\title{
Targeting CXC motif chemokine receptor 4 inhibits the proliferation, migration and angiogenesis of lung cancer cells
}

\author{
WEI HE ${ }^{1}$, TONG YANG ${ }^{2}$, XIN-HUA GONG ${ }^{3}$, RU-ZHAI QIN $^{1}$, XIAO-DONG ZHANG $^{1}$ and WEN-DAN LIU ${ }^{1}$ \\ Departments of ${ }^{1}$ Medical Oncology, ${ }^{2}$ Pathology and ${ }^{3}$ General Medicine, \\ The Second Affiliated Hospital of Guangzhou Medical University, Guangzhou, Guangdong 511447, P.R. China
}

Received November 9, 2017; Accepted April 20, 2018

DOI: $10.3892 / \mathrm{ol} .2018 .9076$

\begin{abstract}
An increasing volume of data indicates that disrupting the interaction between $\mathrm{CXC}$ motif chemokine receptor 4 (CXCR4) and its specific ligand, CXC motif chemokine 12 (CXCL12), may reduce tumor growth and metastasis. However, the translation from bench to bedside must be performed with extreme caution, as the CXCR4/CXCL12 axis is crucial for the normal development and maintenance of tissues and organs. In the present study, Cell Counting Kit-8 and Transwell migration assays were used to detect in vitro proliferation and chemotaxis of CXCR4-expressing A549 cells, a cell strain originating from human non-small-cell lung cancer (NSCLC), with or without the presence of AMD3100, a small-molecule inhibitor specific to CXCR4 signaling. In a xenograft model established by injecting nude mice with A549 cells, tumor growth, CXCR4 expression and microvessel density (MVD) in the tumor mass were determined through tumor size measurements and immunohistochemical staining following intraperitoneal administration of AMD3100 or vehicle. The results demonstrated that CXCR4 blockade inhibited the proliferation of A549 cells and their migration towards CXCL12 in vitro. Tumor growth, CXCR4 expression and MVD were markedly reduced in nude mice treated with AMD3100 compared with mice treated with the vehicle. In conclusion, the present data demonstrated that CXCR4 targeting impaired NSCLC cell growth, angiogenesis and metastatic spread, indicating that it may represent a novel treatment strategy for NSCLC.
\end{abstract}

Correspondence to: Dr Wei He, Department of Medical Oncology, The Second Affiliated Hospital of Guangzhou Medical University, 63 South Yayun Road, Panyu, Guangzhou, Guangdong 511447, P.R. China

E-mail: heman1974@163.com

Key words: CXC motif chemokine receptor 4, non-small-cell lung cancer, cell proliferation, cell migration, angiogenesis, CXC motif chemokine 12

\section{Introduction}

Lung cancer is the leading cause of cancer-associated mortality worldwide in males and females. Non-small-cell lung cancer (NSCLC) accounts for $\sim 85 \%$ of all lung cancer cases $(1,2)$. Despite continuous endeavors to develop novel treatment strategies, the majority of patients with lung cancer exhibit a poor prognosis $(1,2)$. Basic biological behaviors of cancer cells, including migration, angiogenesis and cross-talk between cancer cells and their microenvironment, serve critical roles in tumor growth and metastasis; chemokines are considered to be specific major modulators in these biological processes (3).

Chemokines, also referred to as chemotactic cytokines, are proteins secreted into the blood circulation that mediate numerous physiological and pathological processes primarily associated with cell homing and migration through interactions with the chemokine receptors present on target cells (4). Accumulating data have indicated that tumor growth, invasion and metastasis may be facilitated by the overexpression of chemokine receptors on cancer cells (3), among which CXCR4 is the most extensively investigated. CXCR4, a type of 7-transmembrane $\mathrm{G}$ protein-coupled receptor, is the cognate receptor for stromal cell-derived factor-1 (CXCL12) and is expressed on naive T cells, natural killer cells, dendritic cells and monocytes (5). Recently, it was also identified to be overexpressed in several different types of human cancer, such as breast and pancreatic cancer $(6)$. Meta-analyses $(7,8)$ and clinical $(9,10)$ have demonstrated that CXCR4 expression is associated with organ-specific metastasis and poor survival in patients with NSCLC. An increasing number of studies have elucidated the mechanisms by which chemokine receptors are involved in cancer progression, resulting in the design of corresponding antagonists (11), several of which have already been identified to be effective in certain tumor types according to preliminary $(12,13)$. However, the data are conflicting and the knowledge is incomplete regarding the complexity and diversity of the biological effects induced by CXCR4-CXCL12 (3). Additional studies are required to elucidate these interactions in different types of human cancer prior to clinical application.

In the present study, A549, a cell strain originating from human NSCLC, was used as an in vitro model. The interaction of CXCR4 with CXCL12 was blocked by a CXCR4 antagonist, AMD3100, followed by evaluation of A549 cell proliferation and migration towards CXCL12 with Cell Counting Kit-8 
(CCK-8) and Transwell migration assays. In a preclinical model developed by inoculating nude mice with A549 cells, tumor size and microvessel density (MVD) were compared between experimental and control mice. In addition, the effect of AMD3100 administration on CXCR4 expression was also determined by joint evaluation of the extent and intensity of immunohistochemical (IHC) staining. The results of the present study demonstrated targeting CXCR4 decreased the proliferation, migration and angiogenesis of lung cancer cells.

\section{Materials and methods}

Reagents. A CCK-8 kit was purchased from Dojindo Molecular Technologies, Inc. (Rockville, MD, USA). The Transwell chamber was purchased from Corning Life Sciences (Corning, NY, USA). AMD3100 was supplied by MedChem Express (cat. no. HY-10046; Monmouth Junction, NJ, USA) and dissolved in dimethyl sulfoxide (DMSO) for the cell assay and in sterile PBS for animal administration. Recombinant human CXCR12 protein was purchased from R\&D Systems, Inc. (cat. no. 350-NS; Minneapolis, MN, USA). Monoclonal rabbit anti-human CXCR4 antibody was provided by Abcam (cat. no. ab181020; Cambridge, UK). Mouse anti-human CD34 monoclonal antibody (cat. no. GM716502), horseradish peroxidase (HRP)-labeled anti-rabbit/mouse secondary antibody (cat. no. GP016129) and DAB coloring agent (cat. no. GK500510A) were all purchased from Gene Tech Biotechnology Co. Ltd. (Shanghai, China).

Animals and cell lines. The A549 human alveolar adenocarcinoma cell line was a gift from Professor Liu Ming (Guangzhou Institute of Respiratory Diseases, Guangzhou, China) and maintained in RPMI-1640 (Hyclone; GE Healthcare Life Sciences, Logan, UT, USA) supplemented with $10 \%$ fetal bovine serum (FBS; Gibco; Thermo Fisher Scientific, Inc., Waltham, MA, USA, cat. no. 10099-141), $100 \mathrm{U} / \mathrm{ml}$ penicillin and $100 \mu \mathrm{g} / \mathrm{ml}$ streptomycin at $37^{\circ} \mathrm{C}$ in a $5 \% \mathrm{CO}_{2}$ incubator. Female BALB/c nude mice ( $\mathrm{n}=10$, 4-6 weeks old, 15-18 g) were provided by Beijing Vital River Laboratory Animal Technology Company (Beijing, China) and housed under specific pathogen-free conditions at $25 \pm 2^{\circ} \mathrm{C}$ in a $12 \mathrm{~h}$ night/dark cycle. All animals were fed sterilized rodent food, and has unrestricted access to food and water. They were cared for in accordance with the Animal Welfare Act guidelines under an animal protocol approved by Guangzhou Medical University Animal Care and Use Committee. For experimental end-points, tumor-bearing mice were euthanized prior to the subcutaneous tumor reaching $20 \mathrm{~mm}$ in any direction, but all animals exhibiting signs including restriction of mobility, the inability to feed, pressure on internal organs or sensitive regions of the body or a body condition score (14) of $<2$ were euthanized, even if the maximum tumor size had not been reached.

Cell proliferation assay. The cell proliferation assay was performed using a CCK- 8 kit, according to the manufacturer's protocol. Briefly, A549 cells grown to $80 \%$ confluence were rinsed with $0.25 \%$ Trypsin/EDTA solution (Thermo Fisher Scientific Inc.,) and $2 \mathrm{ml}$ of trypsin solution was added to detach the cells. The detachment was monitored under an inverted microscope for 2-5 min at a magnification of x100. A total of
$8 \mathrm{ml}$ of RPMI 1640 complete medium were added to cease cell detachment and cells were washed twice with phosphate-buffered saline (PBS) followed by centrifugation at $4^{\circ} \mathrm{C}, 100 \mathrm{xg}$ for $5 \mathrm{~min}$. The cells were suspended in complete medium and plated in 96-well plates at a density of 10,000 cells/well and cultured for $12 \mathrm{~h}$ in RPMI-1640 growth medium containing $10 \% \mathrm{FBS}, 100 \mathrm{U} / \mathrm{ml}$ penicillin and $100 \mu \mathrm{g} / \mathrm{ml}$ streptomycin at $37^{\circ} \mathrm{C}$ in a $5 \% \mathrm{CO}_{2}$ incubator. The culture supernatant was removed and growth medium with AMD3100 was added to a final concentration of $2 \mu \mathrm{mol} / 1$ for an additional $0,24,48$, 72 or 96 h culture. An equal volume of growth medium with vehicle alone was added to the control wells. Each medium was added to triplicate wells. Prior to the end of the culture (4 h), AMD3100-containing medium was replaced with $110 \mu \mathrm{l}$ growth medium containing $10 \mu \mathrm{l}$ CCK-8 solution. Following a $4 \mathrm{~h}$ incubation, the optical density (OD) of each well was determined at $450 \mathrm{~nm}$ with an enzyme-linked immunometric meter (BIOBASE-EL10A, BioBase, Jinan, China), and the average OD values were calculated from triplicate wells. The experiment was repeated 3 times independently.

Chemotaxis assay. A549 cells grown to $80 \%$ confluence were harvested as described previously and resuspended in growth medium composed of $2 \mu \mathrm{mol} / 1$ AMD3100 under the aforementioned culture conditions. The cells treated with vehicle alone (DMSO) were used as the control. After $48 \mathrm{~h}$, all cells were serum-starved and cultured for $12 \mathrm{~h}$, followed by assessing chemotaxis to CXCL12. A chemotaxis assay was performed in triplicate in 24-well Transwell chambers with polycarbonate membranes of $8-\mu \mathrm{m}$ pore size. Briefly, $2 \times 10^{4}$ A549 cells in $100 \mu 1$ serum-reduced RPMI-1640 medium containing $1 \%$ bovine serum albumin (MedChemExpress) were added to the upper chamber of each well, and $700 \mu \mathrm{l}$ RPMI-1640 medium containing $10 \% \mathrm{FBS}$ and $10 \mathrm{ng} / \mathrm{ml}$ soluble CXCL12 was added to the lower chamber. Chemotaxis was allowed at $37^{\circ} \mathrm{C}$ in a $5 \%$ $\mathrm{CO}_{2}$ incubator for $12 \mathrm{~h}$. Cells adhering to the upper surface of the membrane were removed with a cotton swab. Cells that had migrated through the filter and adhered to the lower surface of the membrane were fixed by $4 \%$ paraformaldehyde at room temperature (RT) for $30 \mathrm{~min}$ and stained with $0.1 \%$ crystal violet solution at RT for min. Following continuous washing 3 times with running water for $5 \mathrm{~min}$ each, the membranes were removed from the Transwell inserts and Image-Pro Plus software (Ver. 6.0, Media Cybernetics, Inc., Rockville, MD, USA) was used to count the cells under the light microscope (Olympus BX53; Olympus Corporation, Tokyo, Japan) in 6 randomly selected fields at a magnification of x400.

Nude mice lung cancer model. For the xenograft model, $1 \times 10^{6}$ A549 cells were inoculated subcutaneously in the right flank of each BALB/c nude mouse. The length and width of the subcutaneous tumor were measured regularly and the tumor volumes were calculated using the formula: Volume $=\left(\right.$ length $\mathrm{x}$ width $\left.^{2}\right) / 2$. Tumors were not permitted to exceed $2 \mathrm{~cm}$ in diameter; all mice only developed one subcutaneous tumor. Once the tumor width had reached $0.5 \mathrm{~cm}$, the mice were randomly divided into two groups ( $n=5 /$ group). The mice in the two groups received intraperitoneal administration of either AMD3100 (1.25 mg/kg body weight; treatment group) or vehicle (100 $\mu 1$ sterile PBS; control group) twice daily 
for 20 (15). The tumor size was measured every other day. At day 19 when the difference in tumor size became marked in the two groups, all mice were euthanized by exposure to an overdose of $\mathrm{CO}_{2}$, at a flow rate of $20 \%$ of the chamber volume per minute. The tumor xenografts and lung tissues were excised and fixed in $4 \%$ buffered paraformaldehyde at RT for $30 \mathrm{~min}$, and embedded in paraffin.

IHC staining for CXCR4 and CD34 expression. Staining procedures were performed according to the antibody manufacturer's protocol. Briefly, tissues embedded in paraffin were cut into $4-\mu \mathrm{m}$ sections, deparaffinized and rehydrated in a graded alcohol series $(100,95,80$ and $70 \%$, for 1 min each at RT), prepared from absolute alcohol (cat. no. 64-17-5; Shanghai Macklin Biochemical Co., Ltd., Shanghai, China). Following antigen retrieval in sodium citrate buffer $(10 \mathrm{mM}, \mathrm{pH} 6.0$; Haoran Bio-Pharma Co., Ltd., Shanghai, China), endogenous peroxidase was blocked by $3 \% \mathrm{H}_{2} \mathrm{O}_{2}$ in methanol for $10 \mathrm{~min}$ at RT. Tissue sections were then blocked with normal goat serum (cat. no. ab7481; Abcam) for $30 \mathrm{~min}$ at $37^{\circ} \mathrm{C}$ and then incubated with rabbit anti-human CXCR4 (1:400 dilution) or mouse anti-human CD34 (1:200 dilution) at $4^{\circ} \mathrm{C}$ overnight. Subsequent to washing (1X PBS, three times, 5 min each), the tissue sections were incubated with HRP-labeled goat anti-rabbit/mouse antibody (1:100 dilution) at $37^{\circ} \mathrm{C}$ for $15 \mathrm{~min}$. The tissue sections were incubated with the freshly prepared DAB coloring agent (50-100 $\mu \mathrm{l}$ ) at room temperature for 3-10 min, examined under a microscope (Olympus BX53; Olympus Corporation, Tokyo, Japan) at a magnification of $\mathrm{x} 200$ and counterstained with $0.2 \%$ hematoxylin for $2 \mathrm{~min}$ at RT.

Scoring of CXCR4 expression. CXCR4 expression was quantified as previously described $(16,17)$, with modifications. In brief, two scoring systems were used to quantify CXCR4 staining. The percentage of positive tumor cells was referred to as staining extent $(\mathrm{E})$, which was graded on a scale of $0-3$ as follows: $0, \leq 5 \% ; 1,6-20 \% ; 2,21-50 \%$; and 3, >50\%. Staining intensity (I) was also graded on a scale of 0-3 as follows: 0 , none; 1 , weak staining $(+) ; 2$, moderate staining $(++)$; and 3, strong staining $(+++)$. The product of $\mathrm{E} \mathrm{x} \mathrm{I,} \mathrm{referred} \mathrm{to} \mathrm{as} \mathrm{the}$ EI score, ranged from 0 to 9 , and was used to quantify the CXCR4 expression of each section. In view of the heterogeneous expression of CXCR4 in tumor tissues, the total EI score for any section was calculated as a sum of EI scores for 2-3 different fields of view

Microvessel counting. Microvessel counting was performed as previously described (18) under a microscope (at x40 and x100 magnification, DM2500, Leica Microsystems Inc.,Wetzlar, Germany) and pictures were taken by a color camera (TK-C9501EC, Victor Company of Japan, Limited). In brief, the slides were examined at low magnification (x40 and x100) to identify the highest vascular density areas (hot spots) within the tumor, and then 3-4 areas of highest neovascularization were selected for counting at a magnification of x200 (0.739 $\mathrm{mm}^{2} /$ field $)$. The mean number of microvessels of these areas was recorded as the MVD level. Any brown-stained endothelial cell or endothelial cell cluster that was clearly separate from adjacent microvessels, tumor cells and other connective tissue elements, was considered as a single countable microvessel. Vessel lumina and red blood cells were not necessary for defining a microvessel. Macrovessels, characterized by thick muscular walls or with lumina $>8$ red blood cells in diameter $(\sim 50 \mu \mathrm{m})$, were excluded from the count.

Statistical analysis. All data were presented as mean \pm standard error of the mean. GraphPad Prism v.5.01 (GraphPad Software, Inc., La Jolla, CA, USA) software was used for graph plotting and statistical analysis. Relative cell viability was compared using a two-way analysis of variance, followed by Bonferroni's multiple comparison test. The Mann-Whitney U test was used for comparison between two means. $\mathrm{P}<0.05$ was considered to indicate a statistically significant difference.

\section{Results}

CXCR4 blockade effectively inhibits in vitro proliferation of A549 cells and their migration towards CXCL12. AMD3100, a selective small-molecule inhibitor of CXCR4 signaling, was added into the culture medium of A549 cells, and cell viability and migration were observed. As shown in Fig. 1A, cell viability in the control group increased rapidly with increases in culture time and until the endpoint of incubation (>6-fold enhancement in relative cell viability). Regarding the AMD3100-treated group, relative cell viability exhibited a markedly slower increase, followed by a decline $96 \mathrm{~h}$ later $(\mathrm{P}<0.05)$. At the end of the incubation, the cell viability was increased by a little more than 1-fold. CXCR4-expressing A549 cells migrate towards their specific ligand, CXCL12 (6); this phenomenon is referred to as chemotaxis. However, when blocked by AMD3100, the number of A549 cells that migrated through the chamber membrane was markedly reduced compared with vehicle control ( $\mathrm{P}=0.001$; Fig. 1B). The crystal violet staining demonstrated that cells that had migrated through the filter and adhered to the lower surface of the membrane were increased in the vehicle group (Fig. 1C) compared with the AMD3100 group (Fig. 1D)

CXCR4 blockade decelerates tumor growth in nude mice with NSCLC. To determine whether AMD3100 was able to decelerate tumor growth in vivo, a NSCLC xenograft model was established by subcutaneously injecting nude mice with human A549 cells. Mice bearing xenograft tumors were treated with AMD3100 or vehicle, and the tumor volumes were measured. At the endpoint of the study (Day 19), all mice were sacrificed and larger tumor masses were macroscopically observed in vehicle-treated mice than AMD3100-treated mice (Fig. 2A). In addition, tumor growth curve showed that tumor growth was significantly slower in the AMD3100-treated mice compared with the vehicle group ( $\mathrm{P}=0.0247$; Fig. $2 \mathrm{~B})$.

CXCR4 expression and vascularization are inhibited by an antagonist. Tumor xenografts were removed from euthanized mice and CXCR4 expression in tumor cells and MVD in the tumor mass were additionally analyzed by IHC staining. It was demonstrated that CXCR4 expression was localized to the cell membranes (Fig. 3A and B). Tumor sections from AMD3100-treated mice exhibited significantly decreased total EI scores of CXCR4 expression compared with those from 

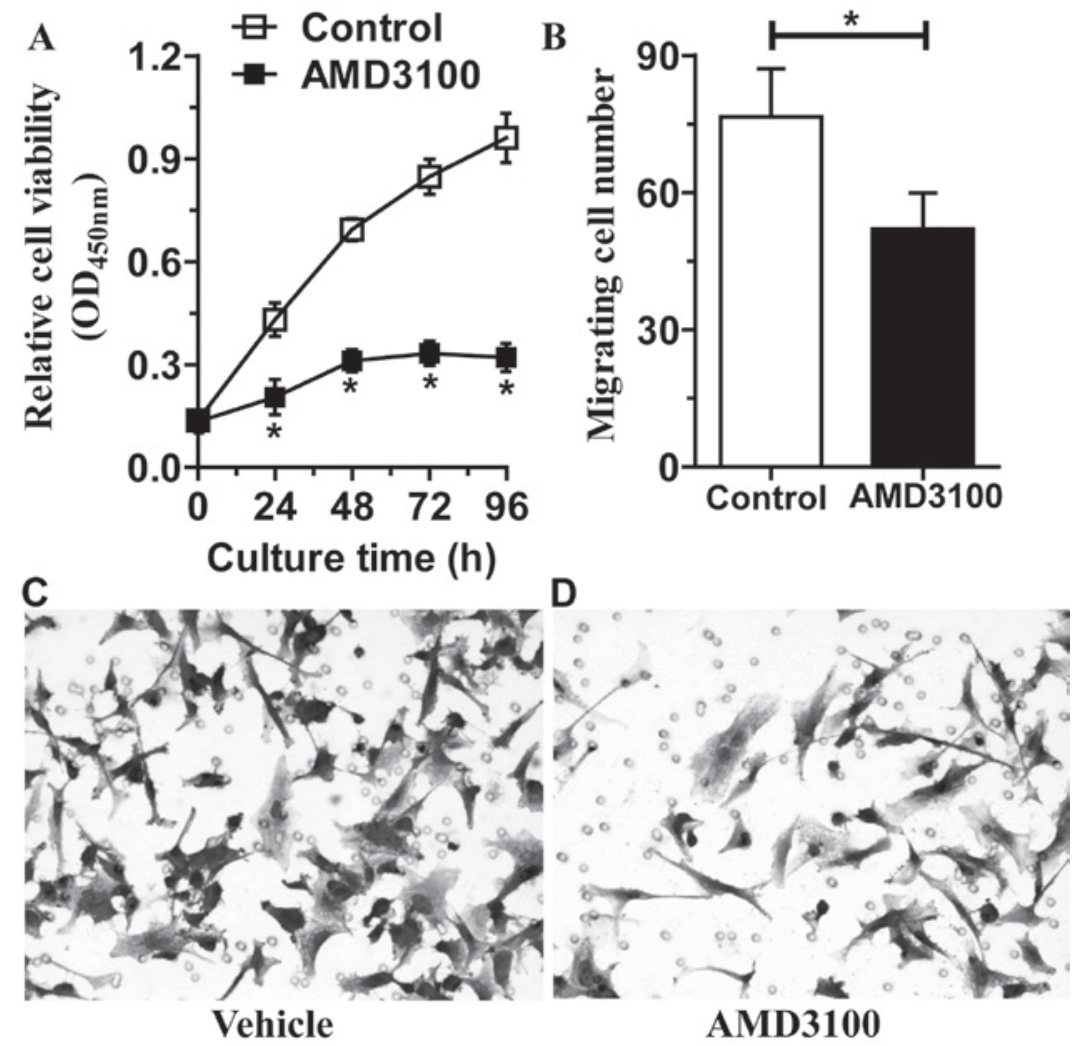

Figure 1. In vitro cell proliferation and migration towards CXCL12 are markedly inhibited by CXCR4 blockade. A549 cells were cultured in vitro with AMD3100 $(2 \mu \mathrm{mol} / 1)$ or its vehicle (DMSO). Data are presented as the mean \pm standard deviation of at least three independent experiments. (A) Relative cell viability at indicated culture times. (B) Cell numbers that migrated through the membrane. Representative crystal violet staining patterns for (C) the vehicle-treated group or (D) the AMD3100-treated group at a magnification of $\mathrm{x} 400$. " $\mathrm{P}<0.05$ vs. vehicle group. OD, optical density; DMSO, dimethyl sulfoxide; CXCR4, CXC motif chemokine receptor 4; CXCL12, CXC motif chemokine 12.
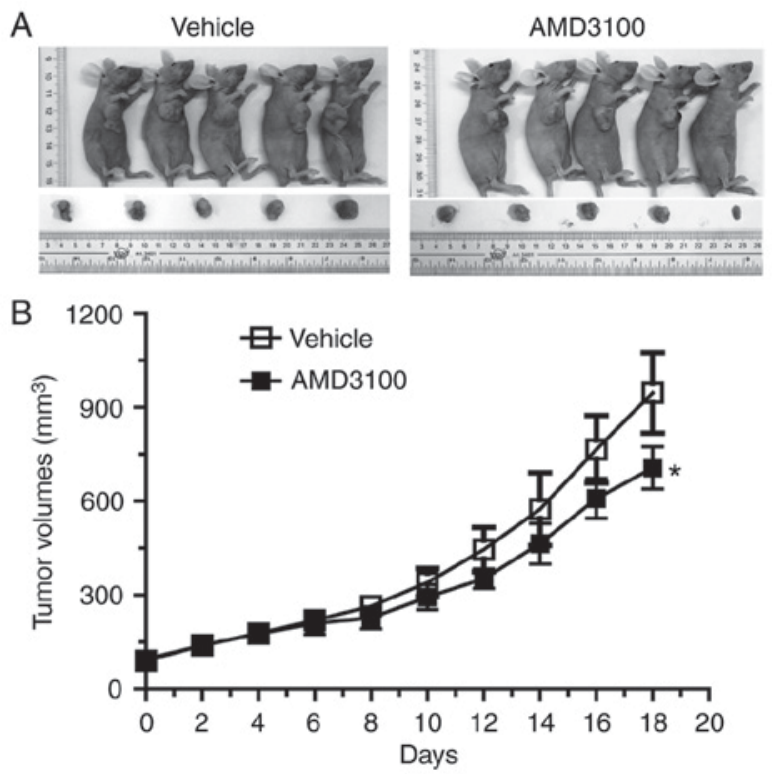

Figure 2. CXCR4 targeting decelerates tumor growth in xenograft mice. A total of $10 \mathrm{BALB} / \mathrm{c}$ nude mice were subcutaneously injected with viable A549 cells and then AMD3100 (1.25 mg/kg body weight) or vehicle (sterile PBS) was administered intraperitoneally twice daily. Each treatment group included 5 mice. The length and width of the subcutaneous tumor mass were measured every other day and tumor volumes were calculated as follows: Volume $=($ length $\mathrm{x}$ width $\left.^{2}\right) / 2$. The results are presented as the mean \pm standard deviation of 5 determinations. (A) Macroscopic evaluation of the size of subcutaneous or excised tumor mass in vehicle-treated (left) or AMD3100-treated (right) mice. (B) Tumor volumes at indicated days. ${ }^{*} \mathrm{P}=0.0247$ vs. vehicle-treated mice. PBS, phosphate-buffered saline. CXCR4, CXC motif chemokine receptor 4. control mice ( $\mathrm{P}=0.0179$; Fig. 3C), the EI score is considered to be positively associated with antigen expression level (16). Subsequently, the microvessels in the tumor mass were visualized by CD34 antigen staining (Fig. 3D and E). By calculating microvessel numbers per $\mathrm{mm}^{2}$ slice, a decreased MVD was observed in AMD3100-treated mice compared with that in control mice $(\mathrm{P}=0.0159$, Fig. $3 \mathrm{~F})$. In summary, these data indicate that CXCR4 targeting is likely to downregulate tumor expression of CXCR4 and impair tumor vascularization.

\section{Discussion}

CXCR4 belongs to the superfamily of $\mathrm{G}$ protein-coupled receptors (GPCRs) that possess seven transmembrane domains (5). CXCR4 is constitutively identified and widely expressed by numerous cell types, including the majority of hematopoietic cell types in the blood and bone marrow, vascular endothelial cells, Langerhans cells, neurons and neuronal stem cells (4). Through binding to CXCL12, which is widely expressed in multiple organs, including the colon, liver, brain, lungs, heart, kidney and spleen, CXCR4 is extensively involved in various physiological functions, including embryonic hematopoiesis, organogenesis, vascularization and immune surveillance, and pathological processes, including inflammation, tumorigenesis and wound healing (4). The roles of chemokine/chemokine receptor interactions in cancer biology have been attracting an increasing amount of attention. As it is overexpressed in $>23$ different types of human cancer (4), CXCR4 is considered 
to contribute to tumor growth, angiogenesis, metastasis and resistance to treatment $(6,12)$. With regards to lung cancer, clinical data indicated that CXCR4 overexpression was significantly associated with poorer progression-free survival time and overall survival rate $(\mathrm{OS})(8,9,19)$. A number of experimental studies demonstrated that in vitro or in vivo inhibition of CXCR4/CXCL12 interaction via CXCR4 blockade $(13,15,20,21)$ attenuated NSCLC cell growth, migration, angiogenesis and metastasis and increased the sensitivity of cancer cells to chemotherapy (22). It appears that this axis may represent a promising target for the development of novel anticancer chemokine-based therapeutics. In fact, multiple agents targeting CXCR4/CXCL12 signaling in cancer are currently being developed (4), among which the CXCR4 antagonist AMD3100 is the most extensively investigated (12). AMD3100, also referred to as plerixafor, was initially studied as an anti-HIV agent, and it was then identified to increase the white blood cell count in the blood and mobilize stem cells from the bone marrow (12). In 2008, AMD3100 gained Food and Drug Administration approval for mobilization of hematopoietic stem cells for bone marrow transplantation, rather than for cancer treatment (23).

Despite the collective aforementioned evidence mentioned supporting the potential efficacy of CXCR4 inhibition for the treatment of cancer, there remains controversy and uncertainty regarding the role of the CXCR4/CXCL12 axis in cancer growth and metastasis. For example, regarding the prognostic value of CXCR4 overexpression, a number of studies concluded that CXCR4 expression was associated with unfavorable prognosis $(8-10,13)$. However, a number of studies also reported different, even opposite conclusions: CXCR4 was detected on the cell membrane, in the cytoplasm and in the nucleus of gastric cancer and NSCLC cells, and strong CXCR4-positive nuclear staining was associated with a significantly improved OS rate $(24,25)$, although the underlying mechanisms remain unclear. In addition, there are data indicating that CXCR4 expression exerted no significant effect on patient survival and was not significantly associated with any other clinicopathological variables (26). Furthermore, increased levels of CXCR4 expression in the tumor cells in comparison with tumor cells with no or low levels of CXCR4 expression from the patients with lung adenocarcinoma, were even identified to be an independent predictor of an improved prognosis in patients with lung adenocarcinoma (27), whereas epigenetic silencing of CXCR4 expression facilitated metastasis and progression of cervical cancer by causing loss of cell-to-cell adhesions (28). Additionally, contradictory data have also been demonstrated regarding the association between CXCL12 expression and clinical outcome $(26,29)$. Despite laboratory data supporting the inhibition of tumor growth and metastasis by CXCL12 expression (30,31), it is reasonable to hypothesize that the CXCR4/CXCL12 axis may exhibit an important antitumor role under certain conditions (32). Discrepancies in observations indicate that our knowledge of certain aspects of this chemokine axis remains incomplete, and additional investigations are required. The therapeutic strategies that block CXCR4 signaling must undergo continued evaluation prior to clinical application, due to the ubiquitous expression of CXCR4 in normal tissues and the functional importance of CXCR4-CXCL12 interaction.
In our previous study (33), 61 patients with NSCLC were analyzed with IHC staining for CXCR4 expression. An increased CXCR4 expression rate was observed in tumor tissues compared with normal lung tissue and a positive association of CXCR4 expression with tumor stage and lymph node metastasis was identified. Despite the lack of data on the association between CXCR4 expression and prognosis, it is well-established that a more advanced tumor stage is associated with poorer survival (9). In the present study, the data indicated that CXCR4 blockade markedly attenuated in vitro cell proliferation and migration towards CXCL12, which was consistent with the aforementioned observations described by a number of studies (4,11-13). In addition, in the present study a xenograft tumor model was established to mimic patients with NSCLC. Following continuous intraperitoneal administration of a CXCR4 antagonist, tumor growth in nude mice was demonstrated to be markedly decreased compared with control mice due to the pronounced inhibition of CXCR4 expression and neovascularization in the tumor mass.

It was demonstrated previously that CXCR4 engagement facilitates cancer cell proliferation, survival, angiogenesis and migration by stimulating multiple downstream signaling pathways, including the phosphatidylinositol-3-kinase (PI3K) and mitogen-activated protein kinase (MAPK) pathways (12). AMD3100 prevented CXCR4 from binding to CXCL12 by occupying the ligand-binding pocket, thereby blocking the generation and downward transduction of activation signals (12). Cancer cell migration was previously identified as one of the key steps of distant cancer spread (34). CXCR4-expressing lung cancer cells migrated to tissues or organs with a high level of CXCL12, including lymph nodes, contralateral lung, liver and brain, finally forming metastatic loci $(10,34)$. The CXCR4 antagonist was previously demonstrated to attenuate tumor metastasis by disrupting the interaction between CXCR4 and CXCL12 (15). Of note, the current immunostaining data demonstrated that CXCR4 blockade significantly downregulated CXCR4 expression by tumor tissues. Based on this result, it is reasonable to infer that decreased CXCR4 expression resulted in weakened CXCR4-CXCL12 interaction and resultant decreased metastasis. At present, little is known about how AMD3100 downregulates CXCR4 expression. Theoretically, CXCR4-associated downstream signaling pathways, including PI3K/Akt, MAPK and Janus kinase/Signal transducer and activator of transcription, all promote anti-apoptosis; therefore, apoptosis induction by signal inhibition is likely to be involved in this downregulation effect. In fact, previous data demonstrated that AMD3100 resulted in increased tumor apoptosis and necrosis (35), although knockdown of CXCR4 by small interfering RNA did not affect tumor cell apoptosis induced by chemotherapeutic agents (36). In the present study, large necrotic areas were observed in tumor tissue sections from mice treated with AMD3100 (data not shown), however the apoptosis data were not obtained and this result requires additional investigation. In addition, it must be mentioned that in the present study CXCR4 expression was only localized to cell membranes, which was not consistent with previous studies $(24,25)$. In the experiment with human NSCLC tissues, positive CXCR4 staining was not observed in the nucleus, but in the cell membrane or the cytoplasm. This result may partly 


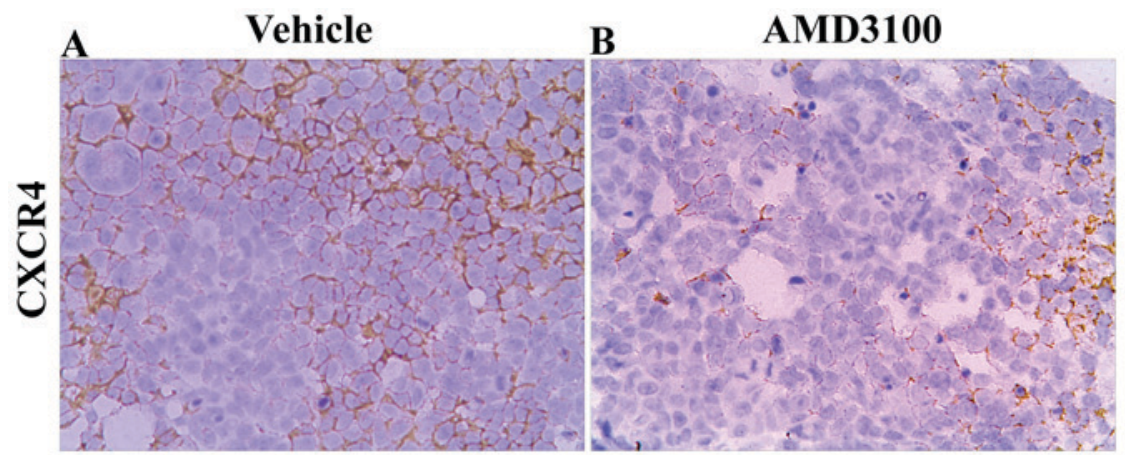

C
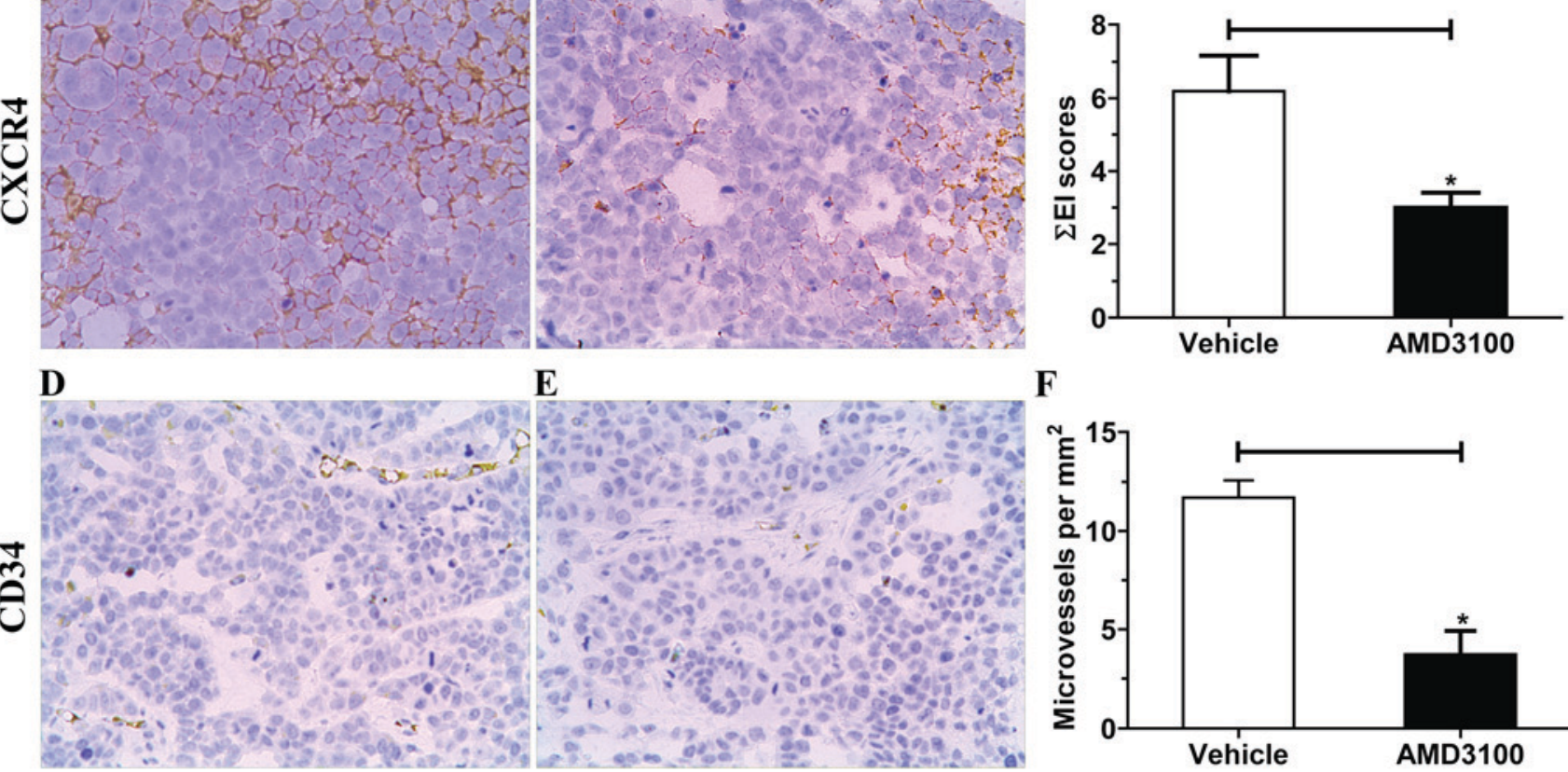

F

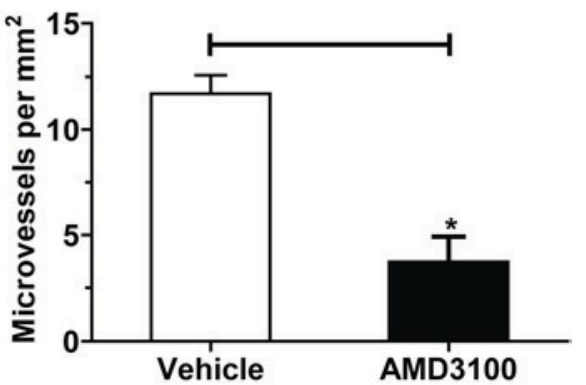

Figure 3. CXCR4 blockade markedly downregulates the expression of CXCR4 and reduces the microvessel density in tumor masses. All mice were sacrificed, then xenograft tumors were removed and subjected to immunohistochemical staining. Anti-CXCR4 staining was used to visualize CXCR4 expression and anti-CD34 staining was used to visualize microvessels. Representative staining patterns for CXCR4 expression in the tumors of mice from (A) the vehicle and (B) the AMD3100 group at a magnification of x200. (C) The total EI scores for CXCR4 expression in the tumors of mice from the two groups. Representative staining patterns of CD34 antigen expression in the tumors of (D) vehicle-treated and (E) AMD3100-treated mice at a magnification of x200. (F) Microvessel numbers per $\mathrm{mm}^{2}$ of tumor tissue from mice in the two groups. " $\mathrm{P}<0.05$ vs. vehicle-treated mice. E, staining extent; I, staining intensity; EI score, E + I scores; CXCR4, CXC motif chemokine receptor 4; CD, cluster of differentiation.

explain our conclusion that high CXCR4 expression was associated with advanced tumor stage, in that patients with nuclear CXCR4 expression were identified to have an improved OS rate compared with those with membranous or cytoplasmic expression (24). Neovascularization is key process in cancer survival and metastatic spread $(15,18)$. The CXCR4/CXCL12 axis was recognized as an important modulator of the angiogenesis/angiostasis balance (37). MVD, a measure of tumor angiogenesis, was reported by various studies $(18,38,39)$ to be closely associated with the incidence of metastases and clinical outcome. By means of immunostaining against the CD34 antigen, a sensitive biomarker for blood vessels, the present study demonstrated a marked reduction of MVD in the tumor masses following treatment with the CXCR4 antagonist. Based on this result, a similar conclusion may be drawn that the CXCR4 blockade may decrease metastasis through inhibition of angiogenesis, in accordance with the findings of previous studies $(11,15)$.

In summary, the data of the present study lead to the conclusion that CXCR4 expression facilitates tumor growth and metastasis, and its targeting may induce antitumor effects to a certain extent by impairing tumor proliferation, migration and angiogenesis. However, despite the encouraging results of the present study and other preclinical data, it is too early to draw definitive conclusions on the role of the CXCR4/CXCL12 axis and its inhibition for cancer treatment. Increased efforts should be focused on elucidating conflicting aspects and improving the understanding of this axis, in order that targeted therapies may be safely applied in clinical settings.

\section{Acknowledgements}

The authors would like to thank Guangzhou Vipotion Biotechnology Company, Limited for the technical support.

\section{Funding}

The present study was supported by the Medical Scientific Research Foundation of Guangdong Province, China (grant no. A2015375 to Dr Wei He).

\section{Availability of data and materials}

The datasets used and/or analyzed during the current study are available from the corresponding author on reasonable request.

\section{Authors' contributions}

WH conceived the experimental design and wrote the manuscript, and WH, TY, XHG, RZQ, XDZ and WDL conducted the experiments. WH and TY analyzed the obtained data. All authors read and approved the final manuscript.

\section{Ethics approval and consent to participate}

Animal experiments were performed in accordance with the Animal Welfare Act guidelines under an animal protocol approved by Guangzhou Medical University Animal Care and Use Committee (Guangzhou, China). 


\section{Patient consent for publication}

Not applicable.

\section{Competing interests}

The authors declare no competing interests.

\section{References}

1. Siegel R, Naishadham D and Jemal A: Cancer statistics, 2012. CA Cancer J Clin 62: 10-29, 2012.

2. Siegel R, DeSantis C, Virgo K, Stein K, Mariotto A, Smith T, Cooper D, Gansler T, Lerro C, Fedewa S, et al: Cancer treatment and survivorship statistics, 2012. CA Cancer J Clin 62: 220-241, 2012.

3. Roy I, Evans DB and Dwinell MB: Chemokines and chemokine receptors: Update on utility and challenges for the clinician. Surgery 155: 961-973, 2014.

4. Chatterjee S, Behnam Azad B and Nimmagadda S: The intricate role of CXCR4 in cancer. Adv Cancer Res 124: 31-82, 2014.

5. Muller A, Homey B, Soto H, Ge N, Catron D, Buchanan ME, McClanahan T, Murphy E, Yuan W and Wagner SN: Involvement of chemokine receptors in breast cancer metastasis. Nature 410 50-56, 2001.

6. Sarvaiya PJ, Guo D, Ulasov I, Gabikian P and Lesniak MS: Chemokines in tumor progression and metastasis. Oncotarget 4 : 2171-2185, 2013

7. Wu Y, Zhang C, Xu W, Zhang J, Zheng Y, Lu Z, Liu D and Jiang K: CXC motif chemokine receptor 4 gene polymorphism and cancer risk. Medicine (Baltimore) 95: e5317, 2016.

8. Zhao H, Guo L, Zhao H, Zhao J, Weng H and Zhao B: CXCR4 over-expression and survival in cancer: A system review and meta-analysis. Oncotarget 6: 5022-5040, 2015.

9. Zhou XM, He L, Hou G, Jiang B, Wang YH and Zhao L: Clinicopathological significance of CXCR4 in non-small cell lung cancer. Drug Des Devel Ther 9: 1349-1358, 2015.

10. Wang L, Wang Z, Liu X and Liu F: High-level C-X-C chemokine receptor type 4 expression correlates with brain-specific metastasis following complete resection of non-small cell lung cancer. Oncol Lett 7: 1871-1876, 2014.

11. Wald O, Shapira OM and Izhar U: CXCR4/CXCL12 axis in non small cell lung cancer (NSCLC) pathologic roles and therapeutic potential. Theranostics 3: 26-33, 2013.

12. Liu T, Li X, You S, Bhuyan SS and Dong L: Effectiveness of AMD3100 in treatment of leukemia and solid tumors: From original discovery to use in current clinical practice. Exp Hematol Oncol 5: 19, 2016

13. Brennecke P, Arlt MJ, Campanile C, Husmann K, Gvozdenovic A, Apuzzo T, Thelen M, Born W and Fuchs B: CXCR4 antibody treatment suppresses metastatic spread to the lung of intratibial human osteosarcoma xenografts in mice. Clin Exp Metastasis 31: 339-349, 2014

14. Ullman-Cullere $\mathrm{MH}$ and Foltz CJ: Body conditioning scoring: A rapid and accurate method for assessing health status in mice. Lab Anim Sci 49: 319-323, 1999.

15. Sun X, Charbonneau C, Wei L, Yang W, Chen Q and Terek RM: CXCR4-targeted therapy inhibits VEGF expression and chondrosarcoma angiogenesis and metastasis. Mol Cancer Ther 12: 1163-1170, 2013.

16. Marechal R, Demetter P, Nagy N, Berton A, Decaestecker C, Polus M, Closset J, Devière J, Salmon I and Van Laethem JL: High expression of CXCR4 may predict poor survival in resected pancreatic adenocarcinoma. Br J cancer 5 100: 1444-1451, 2009.

17. Sood AK, Fletcher MS, Gruman LM, Coffin JE, Jabbari S, Khalkhali-Ellis Z, Arbour N, Seftor EA and Hendrix MJ: The paradoxical expression of maspin in ovarian carcinoma. Clin Cancer Res 8: 2924-2932, 2002.

18. Weidner N, Semple JP, Welch WR and Folkman J: Tumor angiogenesis and metastasis--correlation in invasive breast carcinoma. N Engl J Med 324: 1-8, 1991.

19. Liu Y, Wu BQ, Geng H, Xu ML and Zhong HH: Association of chemokine and chemokine receptor expression with the invasion and metastasis of lung carcinoma. Oncol Lett 10: 1315-1322, 2015 .
20. Gil M, Seshadri M, Komorowski MP, Abrams SI and Kozbor D: Targeting CXCL12/CXCR4 signaling with oncolytic virotherapy disrupts tumor vasculature and inhibits breast cancer metastases. Proc Natl Acad Sci USA 110: E1291-E1300, 2013.

21. Choi WT, Yang Y, Xu Y and An J: Targeting chemokine receptor CXCR4 for treatment of HIV-1 infection, tumor progression, and metastasis. Curr Top Med Chem 14: 1574-1589, 2014.

22. Liang S, Peng X, Li X, Yang P, Xie L, Li Y, Du C and Zhang G: Silencing of CXCR4 sensitizes triple-negative breast cancer cells to cisplatin. Oncotarget 6: 1020-1030, 2015.

23. DiPersio JF, Stadtmauer EA, Nademanee A, Micallef IN, Stiff PJ, Kaufman JL, Maziarz RT, Hosing C, Früehauf S, Horwitz M, et al: Plerixafor and G-CSF versus placebo and G-CSF to mobilize hematopoietic stem cells for autologous stem cell transplantation in patients with multiple myeloma. Blood 113: 5720-5726, 2009.

24. Nikkhoo B, Jalili A, Fakhari S, Sheikhesmaili F, Fathi F, Rooshani D, Hoseinpour Feizi MA and Nikzaban M: Nuclear pattern of CXCR4 expression is associated with a better overall survival in patients with gastric cancer. J Oncol 2014: 808012, 2014.

25. Spano JP, Andre F, Morat L, Sabatier L, Besse B, Combadiere C, Deterre P, Martin A, Azorin J, Valeyre D, et al: Chemokine receptor CXCR4 and early-stage non-small cell lung cancer: Pattern of expression and correlation with outcome. Ann Oncol 15: 613-617, 2004.

26. Popple A, Durrant LG, Spendlove I, Rolland P, Scott IV, Deen S and Ramage JM: The chemokine, CXCL12, is an independent predictor of poor survival in ovarian cancer. Br J Cancer 106: 1306-1313, 2012.

27. Minamiya Y, Saito H, Takahashi N, Ito M, Imai K, Ono T, Motoyama S and Ogawa J: Expression of the chemokine receptor CXCR4 correlates with a favorable prognosis in patients with adenocarcinoma of the lung. Lung Cancer 68: 466-471, 2010.

28. Yadav SS, Prasad SB, Das M, Kumari S, Pandey LK, Singh S, Pradhan S and Narayan G: Epigenetic silencing of CXCR4 promotes loss of cell adhesion in cervical cancer. Biomed Res Int 2014: 581403, 2014.

29. Mirisola V, Zuccarino A, Bachmeier BE, Sormani MP, Falter J, Nerlich A and Pfeffer U: CXCL12/SDF1 expression by breast cancers is an independent prognostic marker of disease-free and overall survival. Eur J Cancer 45: 2579-2587, 2009.

30. Roy I, Zimmerman NP, Mackinnon AC, Tsai S, Evans DB and Dwinell MB: CXCL12 chemokine expression suppresses human pancreatic cancer growth and metastasis. PLoS One 9: e90400, 2014.

31. Wendt MK, Johanesen PA, Kang-Decker N, Binion DG, Shah V and Dwinell MB: Silencing of epithelial CXCL12 expression by DNA hypermethylation promotes colonic carcinoma metastasis. Oncogene 25: 4986-4997, 2006.

32. Williams SA, Harata-Lee Y, Comerford I, Anderson RL, Smyth MJ and McColl SR: Multiple functions of CXCL12 in a syngeneic model of breast cancer. Mol Cancer 9: 250, 2010.

33. Gong X, Yang T, Liu W, Liao K and He W: Expression and clinical significance of chemokine receptor CXCR4 in non-small cell lung cancer. J Trop Med 16: 829-831, 2016 (In Chinese).

34. Popper HH: Progression and metastasis of lung cancer. Cancer Metastasis Rev 35: 75-91, 2016.

35. Righi E, Kashiwagi S, Yuan J, Santosuosso M, Leblanc P, Ingraham R, Forbes B, Edelblute B, Collette B, Xing D, et al: CXCL12/CXCR4 blockade induces multimodal antitumor effects that prolong survival in an immunocompetent mouse model of ovarian cancer. Cancer Res 71: 5522-5534, 2011.

36. Choi YH, Burdick MD, Strieter BA, Mehrad B and Strieter RM: CXCR4, but not CXCR7, discriminates metastatic behavior in non-small cell lung cancer cells. Mol Cancer Res 12: 38-47, 2014.

37. Rivas-Fuentes S, Salgado-Aguayo A, Pertuz Belloso S, Gorocica Rosete P, Alvarado-Vasquez N and Aquino-Jarquin G: Role of chemokines in non-small cell lung cancer: Angiogenesis and inflammation. J Cancer 6: 938-952, 2015.

38. Iakovlev VV, Gabril M, Dubinski W, Scorilas A, Youssef YM, Faragalla H, Kovacs K, Rotondo F, Metias S, Arsanious A, et al: Microvascular density as an independent predictor of clinical outcome in renal cell carcinoma: An automated image analysis study. Lab Invest 92: 46-56, 2012.

39. Weidner N, Carroll PR, Flax J, Blumenfeld W and Folkman J: Tumor angiogenesis correlates with metastasis in invasive prostate carcinoma. Am J Pathol 143: 401-409, 1993. 